\title{
HIGHER ORDER TERMS OF ASYMPTOTIC EXPANSIONS FOR SPHEROIDAL EIGENVALUES*
}

\author{
BY \\ WAN-XIAN WANG ${ }^{1}$ \\ Space Astronomy Laboratory, University of Florida, Gainesville, Florida
}

\begin{abstract}
The convergence of the spheroidal eigenvalues $\lambda_{m n}$ strongly depends upon their initial values. At moderate values of the focal parameter $c$ (which is a product of the wave number and the semi-focal distance of spheroid) and intermediate numbers $n(n>m)$, the asymptotic expansions for $\lambda_{m n}$ of both prolate and oblate spheroidal differential equations require additional higher order terms so that a specific initial value of $\lambda_{m n}$ could be confined within the convergence circle and therefore rapidly reach an accurate value. The previously published results of the asymptotic expansions for both prolate and oblate spheroidal eigenvalues have the higher order terms up to $c^{-5}$. The author has added two additional higher order terms $\left(c^{-6}\right.$ and $\left.c^{-7}\right)$ to the asymptotic expansions, greatly improving the convergence of $\lambda_{m n}$ and speeding up the computational process. In addition, the higher order terms for the eigenvalues of the Mathieu differential equation may be readily obtained by using the transformation from the prolate spheroidal differential equation.
\end{abstract}

1. Introduction. In prolate spheroidal coordinate systems, the angular differential equation of wave functions is expressed in the form:

$$
\left(1-\eta^{2}\right) \frac{d^{2} S_{m n}}{d \eta^{2}}-2 \eta \frac{d S_{m n}}{d \eta}+\left(\lambda_{m n}-c^{2} \eta^{2}-\frac{m^{2}}{1-\eta^{2}}\right) S_{m n}=0,
$$

where $\eta$ is the spheroidal angular coordinate with $-1 \leq \eta \leq 1 ; S_{m n}$ and $\lambda_{m n}$ are the spheroidal angular functions and eigenvalues of order $m$ and degree $n$, respectively; $m$ and $n$ are the positive integers with $n \geq m .^{1}$

By introducing a new angular function $u_{m n}$ such that

$$
S_{m n}=\left(1-\eta^{2}\right)^{m / 2} u_{m n}
$$

and transforming the angular coordinates through

$$
\eta=(2 c)^{-1 / 2} x
$$

Eq. (1) is converted to

$$
\left(1-\frac{x^{2}}{2 c}\right) \frac{d^{2} u_{m n}}{d x^{2}}-\frac{(m+1) x}{c} \frac{d u_{m n}}{d x}+\left(K-\frac{x^{2}}{4}\right) u_{m n}=0,
$$

*Received November 11, 1988.

'Space Astronomy Laboratory, 1810 NW 6th Street, Gainesville, Florida 32609.

(C) 1989 Brown University 
where the constant $K$ is defined by

$$
K=\frac{\lambda_{m n}-m(m+1)}{2 c} .
$$

Then by expanding $u_{m n}$ and $K$ in terms of the inverse power of $c$, that is,

$$
\begin{gathered}
u_{m n}=u_{0}+u_{1} c^{-1}+u_{2} c^{-2}+\cdots, \\
K=\alpha_{0}+\alpha_{1} c^{-1}+\alpha_{2} c^{-2}+\cdots,
\end{gathered}
$$

and substituting them in Eq. (4), the expansion coefficients $\alpha_{r}(r=0,1,2, \ldots)$ along with $\lambda_{m n}$ can be calculated. by

In oblate spheroidal coordinate systems, the angular differential equation is given

$$
\left(1-\eta^{2}\right) \frac{d^{2} S_{m n}}{d \eta^{2}}-2 \eta \frac{d S_{m n}}{d \eta}+\left(\lambda_{m n}+c^{2} \eta^{2}-\frac{m^{2}}{1-\eta^{2}}\right) S_{m n}=0,
$$

from which the following transcendental equation may be generated [1]:

$$
\Lambda_{m n}=\frac{Q_{0}^{2}}{\left(\Lambda_{m n}+P_{-1}\right)-} \frac{Q_{-1}^{2}}{\left(\Lambda_{m n}+P_{-2}\right)-} \cdots+\frac{Q_{1}^{2}}{\left(\Lambda_{m n}+P_{1}\right)-} \frac{Q_{2}^{2}}{\left(\Lambda_{m n}+P_{2}\right)-} \cdots,
$$

where Flammer's notation for continued fractions is adopted, and

$$
\begin{gathered}
Q_{s}=\frac{1}{4}(q+2 s-1-m)(q+2 s-1+m), \\
P_{s}=2 s(q+s-2 c), \\
\Lambda_{m n}=\lambda_{m n}+c^{2}-2 q c+\frac{1}{2}\left(q^{2}+1-m^{2}\right) ;
\end{gathered}
$$

in which

$$
\begin{array}{ll}
q=n & \text { for }(n-m) \text { odd } \\
q=n+1 & \text { for }(n-m) \text { even. }
\end{array}
$$

Next, writing $\Lambda_{m n}$ in the asymptotic form of $c$, viz:

$$
\Lambda_{m n}=\sum_{i=1}^{\infty} \ell_{i} c^{-i}
$$

and substituting it in Eq. (7), we can calculate the coefficients $\ell_{i}(i=1,2,3, \ldots)$; then $\lambda_{m n}$ may be obtained from Eq. (7.3).

2. Asymptotic expressions for prolate and oblate spheroidal eigenvalues. J. Meixner [2] derived the asymptotic expressions for both prolate and oblate spheroidal eigenvalues up to the $c^{-5}$ higher order terms. The author has developed two additional higher order terms. The involved calculations become more lengthy and complicated as the order goes higher. 
The resultant prolate spheroidal eigenvalues $\lambda_{m n}$ are:

$$
\begin{aligned}
& \lambda_{m n}=q c-\left(q^{2}+5-8 m^{2}\right) 2^{-3}-q\left(q^{2}+11-32 m^{2}\right) 2^{-6} c^{-1} \\
&- {\left[5\left(q^{4}+26 q^{2}+21\right)-384 m^{2}\left(q^{2}+1\right)\right] 2^{-10} c^{-2} } \\
&- q\left[\left(33 q^{4}+1594 q^{2}+5621\right)-128 m^{2}\left(37 q^{2}+167\right)+2048 m^{4}\right] 2^{-14} c^{-3} \\
&- {\left[\left(63 q^{6}+4940 q^{4}+43327 q^{2}+22470\right)\right.} \\
&\left.-640 m^{2}\left(23 q^{4}+262 q^{2}+147\right)+24576 m^{4}\left(q^{2}+1\right)\right] 2^{-16} c^{-4} \\
&- q\left[\left(527 q^{6}+61529 q^{4}+1043961 q^{2}+2241599\right)\right. \\
&-32 m^{2}\left(5739 q^{4}+127550 q^{2}+298951\right) \\
&\left.+10240 m^{4}\left(71 q^{2}+301\right)-65536 m^{6}\right] 2^{-20} c^{-5} \\
&- {\left[\left(9387 q^{8}+1536556 q^{6}+43711178 q^{4}+230937084 q^{2}+93110115\right)\right.} \\
&-1536 m^{2}\left(2989 q^{6}+112020 q^{4}+648461 q^{2}+270690\right) \\
&\left.+196608 m^{4}\left(175 q^{4}+1814 q^{2}+939\right)-12582912 m^{6}\left(q^{2}+1\right)\right] 2^{-25} c^{-6} \\
&- q\left[\left(175045 q^{8}+38429780 q^{6}+1671177494 q^{4}\right.\right. \\
&\left.\quad+17095651460 q^{2}+27640944461\right)
\end{aligned}
$$

where

$$
q=2(n-m)+1 .
$$

The eigenvalues of the Mathieu differential equation can be easily obtained from Eq. (9) by setting $m= \pm \frac{1}{2}$ and defining $\Lambda$ (the asymptotic values of the characteristic numbers $a_{n-m}$ and $b_{n-m+1}$ ) such that

$$
\Lambda=\lambda_{m n}+\frac{1}{4}-\frac{c^{2}}{2}
$$

namely,

$$
\begin{aligned}
\Lambda= & -2 h^{2}+2 q h-\left(q^{2}+1\right) 2^{-3}-q\left(q^{2}+3\right) 2^{-7} h^{-1}-\left(5 q^{4}+34 q^{2}+9\right) 2^{-12} h^{-2} \\
& -q\left(33 q^{4}+410 q^{2}+405\right) 2^{-17} h^{-3}-\left(63 q^{6}+1260 q^{4}+2943 q^{2}+486\right) 2^{-20} h^{-4} \\
& -q\left(527 q^{6}+15617 q^{4}+69001 q^{2}+41607\right) 2^{-25} h^{-5} \\
& -\left(9387 q^{8}+388780 q^{6}+2845898 q^{4}+4021884 q^{2}+506979\right) 2^{-31} h^{-6} \\
& -q\left(175045 q^{8}+9702612 q^{6}+107779416 q^{4}\right. \\
& \left.\quad+287224296 q^{2}+120298137\right) 2^{-37} h^{-7}+O\left(h^{-8}\right),
\end{aligned}
$$

where

$$
h=\frac{c}{2} \text {. }
$$

It is worth pointing out that two coefficients in the $h^{-5}$ term for the eigenvalues $\Lambda$ derived by Goldstein [3] need to be corrected: $270379 q^{2}$ and 149553 should be $276004 q^{2}$ and 166428 , respectively. 
The resultant oblate spheroidal eigenvalues $\lambda_{m n}$ are:

$$
\begin{aligned}
\lambda_{m n}= & -c^{2}+2 q c-\left(q^{2}+1-m^{2}\right) 2^{-1}-q\left(q^{2}+1-m^{2}\right) 2^{-3} c^{-1} \\
- & {\left[\left(5 q^{4}+10 q^{2}+1\right)-2 m^{2}\left(3 q^{2}+1\right)+m^{4}\right] 2^{-6} c^{-2} } \\
- & q\left[\left(33 q^{4}+114 q^{2}+37\right)-2 m^{2}\left(23 q^{2}+25\right)+13 m^{4}\right] 2^{-9} c^{-3} \\
- & {\left[\left(63 q^{6}+340 q^{4}+239 q^{2}+14\right)-10 m^{2}\left(10 q^{4}+23 q^{2}+3\right)\right.} \\
- & q\left[\left(527 q^{6}+4139 q^{4}+5221 q^{2}+1009\right)+3 m^{4}\left(13 q^{2}+6\right)-2 m^{6}\right] 2^{-10} c^{-4} \\
& \left.\quad-m^{2}\left(939 q^{4}+3750 q^{2}+1591\right)+5 m^{4}\left(93 q^{2}+127\right)-53 m^{6}\right] 2^{-13} c^{-5} \\
- & {\left[\left(9387 q^{8}+101836 q^{6}+205898 q^{4}+86940 q^{2}+3747\right)\right.} \\
& -12 m^{2}\left(1547 q^{6}+9575 q^{4}+8657 q^{2}+701\right) \\
& \left.+6 m^{4}\left(1855 q^{4}+5078 q^{2}+939\right)-12 m^{6}\left(167 q^{2}+85\right)+51 m^{8}\right] 2^{-17} c^{-6} \\
- & q\left[\left(175045 q^{8}+2520820 q^{6}+7568470 q^{4}+5771940 q^{2}+822221\right)\right. \\
& -4 m^{2}\left(95167 q^{6}+847819 q^{4}+1345421 q^{2}+353449\right) \\
& +6 m^{4}\left(44625 q^{4}+205450 q^{2}+114597\right) \\
+ & O\left(c^{-8}\right) .
\end{aligned}
$$

3. Discussion. The developments on two additional higher order terms of the asymptotic expansions for spheroidal eigenvalues (designated as Method 2) cause the initial eigenvalues to rapidly converge to their final eigenvalues at some moderate values of $c$, small numbers $m$, and intermediate numbers $n$, but this is not the case for the asymptotic expansions with the higher order terms up to $c^{-5}$ (designated as Method 1).

For the comparison between these two methods, we have computed $\lambda_{m n}$ at some specific values of $c, m$ and $n$. In the prolate case, we choose $c=17, m=0, n=9$ : Method 1 does not tend to any convergent eigenvalue at all, and Method 2 gives $\lambda_{09}=264.81922 \cdots$. In the oblate case, we choose $c=12, m=0$ and $n=7$ : Method 1 incorrectly yields another value $\lambda_{05}(=-38.938679 \cdots)$ because the initial eigenvalue falls within the convergence circle of $\lambda_{05}$ but not $\lambda_{07}$; however, Method 2 leads to the correct value $\lambda_{07}(=-6.406991 \cdots)$.

Making use of the asymptotic and the ordinary power series expansions containing the higher order terms, we have virtually closed the gap between these two expansions, which was formed from using previous expansions that lacked the necessary higher order terms.

Acknowledgment. The author is grateful for the valuable suggestion from the referee. 


\section{REFERENCES}

[1] C. Flammer, Spheroidal Wave Functions, Stanford University Press, (1957)

[2] J. Meixner, Asymptotische Entwicklung der Eigenwerte und Eigenfunktionen der Differentialgleichungen der Sphäroid-Funktionen und der Mathieuschen Funktionen, Z. Angew. Math. Mech. 28, 304-310 (1948)

[3] S. Goldstein, Mathieu Functions, Trans. Cambridge Philos. Soc. 23, 303-336 (1927) 